\title{
Accuracy Validation of an Image Guided Laparoscopy System for Liver Resection
}

\author{
Stephen Thompson ${ }^{1}$, Johannes $\operatorname{Totz}^{1}$, Yi Song ${ }^{1}$, Stian Johnsen ${ }^{1}$, Danail Stoyanov ${ }^{1}$, Sébastien \\ Ourselin $^{1}$, Kurinchi Gurusamy ${ }^{2}$, Crispin Schneider ${ }^{2}$, Brian Davidson ${ }^{2}$, David Hawkes ${ }^{1}$, \\ Matthew J Clarkson ${ }^{1}$ \\ ${ }^{1}$ Centre for Medical Image Computing, University College London, United Kingdom \\ ${ }^{2}$ Division of Surgery and Interventional Science, UCL Medical School, United Kingdom
}

\begin{abstract}
We present an analysis of the registration component of a proposed image guidance system for image guided liver surgery, using contrast enhanced CT. The analysis is performed on a visually realistic liver phantom and in-vivo porcine data. A robust registration process that can be deployed clinically is a key component of any image guided surgery system. It is also essential that the accuracy of the registration can be quantified and communicated to the surgeon. We summarise the proposed guidance system and discuss its clinical feasibility. The registration combines an intuitive manual alignment stage, surface reconstruction from a tracked stereo laparoscope and a rigid iterative closest point registration to register the intra-operative liver surface to the liver surface derived from CT. Testing of the system on a liver phantom shows that subsurface landmarks can be localised to an accuracy of $2.9 \mathrm{~mm}$ RMS. Testing during five porcine liver surgeries demonstrated that registration can be performed during surgery, with an error of less than $10 \mathrm{~mm}$ RMS for multiple surface landmarks.
\end{abstract}

\section{INTRODUCTION}

The successful implementation of an image guidance system for laparoscopic liver resection has the potential to improve the feasibility of laparoscopic resection for patients with tumours located in surgically challenging locations. If done well, laparoscopic resection can have equivalent curative results to open surgery but with shorter recovery times. ${ }^{1}$

Whilst image guided surgery of the liver is complicated by the motion and deformation of the liver, there are aspects of liver surgery that make it an ideal candidate for image guided surgery. Liver resection involves removing one or more segments of the liver, cutting through and sealing off their blood supply. This process requires a good understanding of the individual liver anatomy and its blood supply. For this reason there are now multiple companies offering image segmentation services that convert contrast enhanced computed tomography (CT) to patient specific volumetric models (for example www.visiblepatient.com and www.mevis.de). Such models can be used for procedure planning or referred to during surgery, for example via a screen ${ }^{2,3}$ or by $3 \mathrm{D}$ printing of the liver. ${ }^{4}$ Using the pre-operative images in this way requires the surgeon to mentally map the model to the visible patient anatomy. In theory, the process can be improved by registering the model to the patient with a computer assisted surgery system, reducing the cognitive load on the surgeon.

Registering the model to the patient with a computer assisted surgery system introduces three problems. The first is actually performing the registration reliably and within a time frame compatible with the surgical work flow. The second problem is determining and demonstrating the system accuracy. The final problem is allowing the surgeon to understand the functioning of the system to enable confirmation that the system has performed correctly for a given case. As registration algorithms become more complex, for example by including deformations, the accuracy achieved should improve. However this may be at the cost of reliability or the ability to accurately gauge the accuracy of a given registration. To date, therefore, image guidance systems for liver surgery have used rigid registration driven by user defined landmarks or surfaces. ${ }^{2}, 3,5$ These systems provide a robust and easily understood registration, however their accuracy is limited, of the order of $10 \mathrm{~mm}$.

Several groups are working on systems to improve the registration accuracy for laparoscopic image guided liver surgery. Several authors, ${ }^{6,7}$ have examined using intra-operatively digitised surfaces to drive biomechanical model based deformation of the liver. Mountney et al. ${ }^{8}$ propose using intra-operative cone beam CT to drive 
a biomechanically informed non rigid registration. They further refine the registration using intra-operative landmarks acquired using simultaneous localisation and mapping (SLAM). These systems have the potential to provide accurate registration, however at present they require a significant interruption to the surgical work flow, and it is difficult to validate the correct working of the algorithm for an individual case.

The system presented in this paper couples a simple rigid body registration with dense surface patches reconstructed intra-operatively from stereo high definition video and localised using an optical tracking system. The system removes the need for manually defining landmarks and avoids the need for contact with the liver surface with a digitising probe, as Kingham et al. ${ }^{2}$ do. Apart from requiring a stereo scope, the only extra equipment required is an optical tracking system, (NDI Polaris Spectra*), which is widely used in image guided interventions and causes minimal disruption in the operating room. The surface patch reconstruction and registration is performed interactively with a clear user interface that allows a technically able user to assess the performance of the system in real time. The individual components are implemented as modules within the NifTK ${ }^{9}$ software platform.

After describing the functioning of the system, this paper presents a validation of the accuracy of the system as measured on a single plastic phantom and 3 porcine data sets. In addition to demonstrating the accuracy of the current rigid registration, this paper also seeks to show the contribution of static and dynamic deformation to the overall error, giving an estimate of the expected performance of more sophisticated registration algorithms currently under development.

Assuming that a stereo laparoscope is available, the system presented here avoids the need for specialist equipment or manual point selection to enable a rigid registration of preoperative data to the laparoscopic video within a surgically useful time (under 5 minutes).

\section{REGISTRATION METHOD}

The focus of this paper is the validation of registration accuracy obtained by the proposed image guidance system. The registration determines the correspondence between a pre-operative model, derived from contrast enhanced CT data, to intra-operative stereo laparoscopic video. The registration method presented here is divided into five steps performed during surgery: surface patch reconstruction, surface patch localisation, surface patch filtering and compositing, registration initialisation, and final registration. In addition, prior to surgery the patient's CT data is segmented into a surface based model and important anatomy defined. Each of these stages is discussed in the following five sections.

\subsection{Pre Operative Processing}

Prior to surgery the patient's CT scan is segmented into a set of anatomical surface model, representing the patients anatomy of interest, (i.e. liver segments, arteries, portal and hepatic veins, gall bladder, and any CT visible tumours). Segmentation is currently performed externally using the Visible Patient service ${ }^{\dagger}$.

\subsection{Surface Reconstruction}

The aim of the surface reconstruction is to determine the geometry of the visible surfaces relative to the laparoscope. Surface reconstruction is performed by matching features between the two channels of the stereo laparoscope and triangulating their position using the known lens geometries. Prior to the surgery the individual channels of the laparoscope are calibrated, as per Zhang, ${ }^{10}$ by recording multiple views of a static chequerboard, then the camera intrinsic parameters (focal lengths, principal points, and 4 distortion coefficients) are calculated. The calibration algorithm, including the calculation of the transform between the right and left laparoscope lenses, is implemented in OpenCV $\mathrm{V}^{\ddagger}$, with a user interface implemented in NifTK. Approximately 50 views of the chequerboard are used during calibration.

During surgery an implementation of Stoyanov et al. ${ }^{11}$ dense surface reconstruction algorithm is used to match features between the left and right channels. We also use a graphics processing unit (GPU) implementation ${ }^{12}$ that

\footnotetext{
${ }^{*}$ www.ndigital.com

${ }^{\dagger}$ www.visiblepatient.com

${ }^{\ddagger}$ www.opencv.org
} 


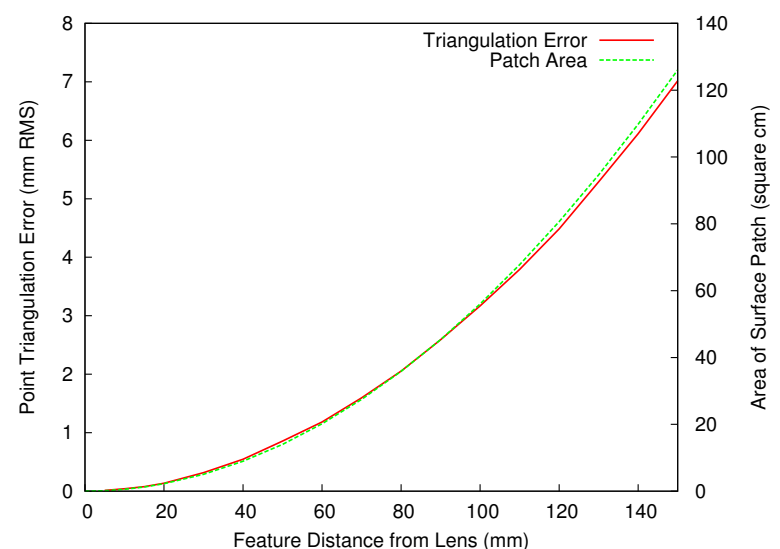

Figure 1. For a stereo camera with a $4.5 \mathrm{~mm}$ baseline, triangulation errors rapidly increase with distance from the lens. Beyond $75 \mathrm{~mm}$ the triangulation is not generally useful.

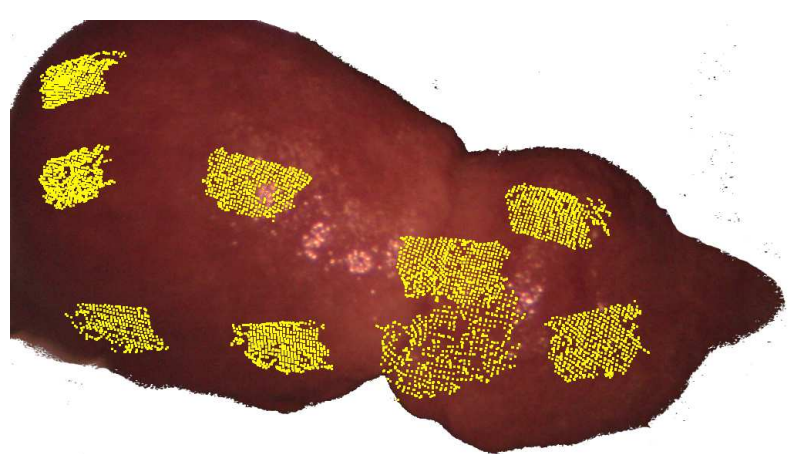

Figure 2. Nine surface patches each with an area around $30 \mathrm{~cm}^{2}$ shown overlaid on the liver phantom.

can perform the matching significantly quicker. At present, however, the performance of the two implementations is very similar due to the overhead in moving image data to and from the GPU. Currently the user interface is set up so that the surgeon first moves the scope to a target area and the surface reconstruction is started via an on screen button. On completion the surgeon is able to visualise the surface patch overlaid on the video, to confirm successful operation. The reconstruction process takes around 2 seconds. At present the system cannot differentiate between the surface of the liver and other anatomical structures. For successful operation the user must ensure that only liver surface is with the patch before commencing reconstruction.

The position of matched features relative to the left lens is determined by projecting rays for each lens and finding the midpoint of the line joining the rays at their point of closest approach. Triangulation is sensitive to the distance between the camera lens (around $4.5 \mathrm{~mm}$ for the Viking 3DHD ${ }^{\S}$ stereo scope used). Figure 1 shows how the triangulation error increases with distance from the laparoscope lens. The practical implication of this is that surfaces can only be triangulated reliably at distances of up to $80 \mathrm{~mm}$ from the lens, at which distance the surface patch is around $30 \mathrm{~cm}^{2}$ in area. Figure 2 shows a visualisation of nine surface patches overlaid on the liver phantom. During phantom and porcine work the proposed algorithm was found to work well when the liver surface was between 50 to $80 \mathrm{~mm}$ from the lens, giving surface patch areas between 14 and $36 \mathrm{~cm}^{2}$. At this scale the human liver is smoothly curved, and cannot be relied on for registration. ${ }^{7,13}$ Therefore it becomes necessary to gather multiple surface patches and combine them.

\subsection{Surface Patch Localisation}

To be useful for registration multiple surface patches must be joined together to form a wider angle model of the liver, therefore it is necessary to determine the location of the surface patches relative to each other. Localisation of patches is done by tracking the external end of the laparoscope using a Polaris Spectra optical tracking system. The set up is shown in figure 3. Prior to surgery the transform between the tracking markers and the laparoscope lens is determined as per Tsai and Reimar, ${ }^{14}$ often referred to as the "handeye" calibration. Each reconstructed surface patch is placed within the coordinate system of the Polaris tracking system. By moving the scope after surface reconstruction the user is able to confirm that the patch is correctly positioned on the visible surface of the liver in real time. The system has been tested during five porcine resections, showing that sufficient (6 to 12) surface patches for registration of a single lobe can be easily collected and checked within 2 minutes.

The patch localisation process depends on correct synchronisation of video and tracking signals. Synchronisation is maintained using the NiftyLink ${ }^{9}$ library to maintain time stamps on all data input streams to NifTK.

\footnotetext{
$\S_{\text {www.conmed.com }}$
} 


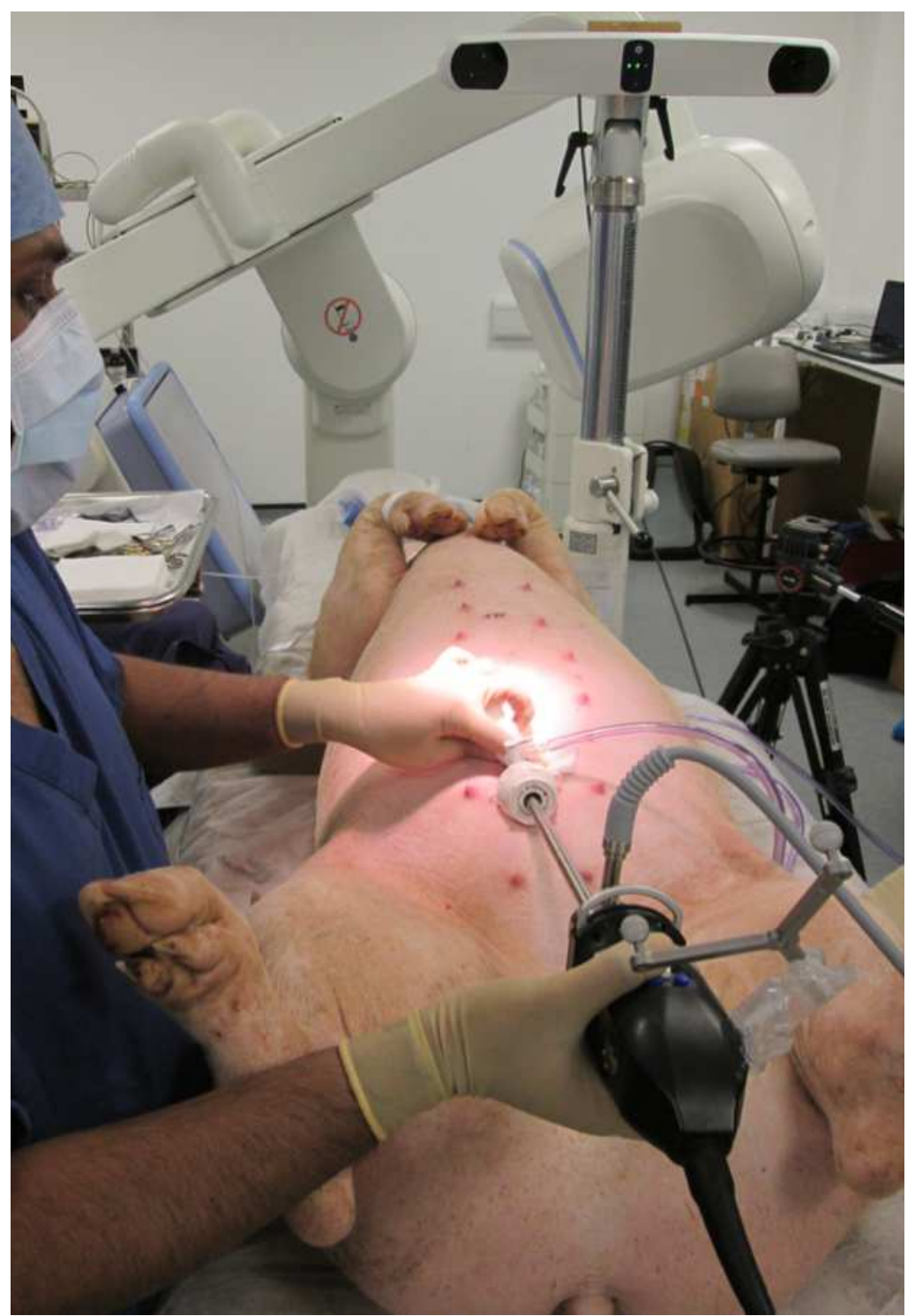

Figure 3. The optical tracking cameras, top right, track a rigid body of three infra red reflective spheres rigidly fixed to the external end of the laparoscope, bottom right. 


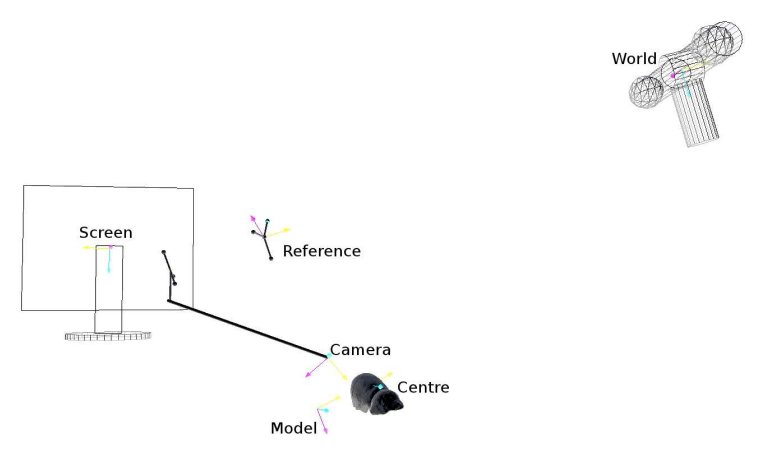

Figure 4. The six coordinate systems used in manual alignment.

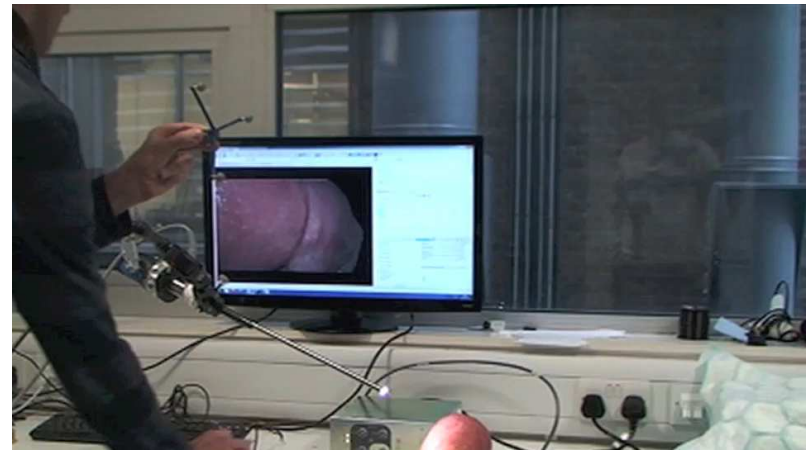

Figure 5. Photo showing the reference rigid body being used to manipulate the on screen model of the liver in the laboratory. An estimate of the transform between screen and tracker system coordinate systems is used to transform all translations and rotations into the coordinate system of the screen.

\subsection{Surface Patch Filtering}

In typical use the reconstructed surface patches consist of between 6 and 12 surface patches, each patch consisting of up to 1 million points. Attempting registration with this quantity of points would be computationally expensive, so a point cloud filtering process is used. The primary purpose of the filtering is to reduce subsequent registration time, the filtering also serves to uniformly re-sample the point clouds so that overlapping point clouds do not unintentionally bias subsequent registration. Filtering re-samples the point cloud by finding the average point location per voxel. Reconstruction noise is also reduced by assuming the points belong to a single surface with a maximum allowable surface curvature, points away from this surface are projected onto it. The filtering is implemented using the point cloud library (PCL),${ }^{15}$ with a graphical user interface implemented in NifTK. Filtering currently takes around 30 seconds.

\subsection{Manual Initialisation of Registration}

At this stage it is possible to register the source image (pre-operative liver surface) to the target image (intraoperative surface patches). How the registration is achieved depends on the data. At present we use the rigid point to surface iterative closest point (ICP) implemented within VTK $\$$. For the phantom, where there is no shape change between source and target, and the surface patches are well spread over the whole liver, the ICP algorithm is relatively insensitive to initialisation and can be run immediately with good results. For the porcine data, however, shape changes between the source and target images and the difficultly in getting well spread surface patches, means that the ICP has many local minima and successful registration is very dependent on an initial registration estimate. Obtaining this estimate in an intuitive and clinically suitable is challenging. We have implemented a novel manual alignment process that enables fast and intuitive alignment whilst maintaining sterility in theatre.

The first stage of the alignment process is to identify the centre of the anatomy of interest in the coordinates of the CT scan. Typically this will be the centroid of the liver lobe undergoing resection. The next stage of alignment relies on the fact that laparoscopic surgery is tightly constrained by patient geometry, i.e. the scope is generally inserted in the lower abdomen looking towards the patients head. Therefore the transform between the scope lens and the anatomy of interest can be estimated prior to surgery commencing. This estimate is generally enough to get both the modelled and the patient's anatomy on the same video screen.

The final stage of manual alignment uses a second rigid body, which can be sterilised if necessary, to "pick up" the virtual liver and move it in the coordinates of the overlay screen. Figures 4 and 5 show the six relevant coordinate systems and a photo of the alignment process. In order to be intuitive we have found that it is useful to transform all rotations and translations into the coordinate system of the screen, rather than the tracking

\footnotetext{
I www.vtk.org
} 
system. Being able to do this depends on knowing the rotational transform from the tracker origin to the screen origin, which will change depending on the set up in theatre. In practice this transform can be set interactively (in less than a minute) once all equipment is set up in theatre.

Once this is done the user/surgeon can use the additional rigid body to move the modelled liver on screen until it overlays the visible anatomy. We have tested this process repeatedly in the laboratory on a plastic phantom and in theatre on porcine models. The system has proven intuitive and robust, we are able to achieve an initial estimate in under 3 minutes.

\subsection{Registration}

The final stage of the registration uses ICP to minimise the distance between the reconstructed surface points and the pre-operatively segmented liver surface. The algorithm currently used is VTK's point to surface implementation.

As porcine livers can be very thin in places we found there were occasions when the reconstructed point clouds matched to the non visible rear surface of the liver. To avoid this a hidden surface removal algorithm was incorporated in the registration so that only surfaces of the liver visible from the current camera position are used for registration. The functioning of the hidden surface removal is obviously dependent on a good starting estimate of the position of the liver relative to the camera, hence the need for a good initial estimate, see Section 2.5 .

The registration itself takes less than 10 seconds to complete. The user interface enables the user to undo any registration, and if necessary try rerunning it from a different starting estimate. We found that this was not necessary for the phantom liver, but several attempts were sometimes needed on the porcine data to get a visually satisfactory registration. This last point highlights the importance of a good interface design, which enables the system to be used and its accuracy assessed in real time during surgery. The results presented in the following sections are based on registrations performed in theatre prior to resection of the porcine liver, not on any post operative retrospective optimisation.

\section{VALIDATION METHOD}

The functioning and accuracy of the system was assessed on a visually and geometrically realistic silicone phantom and on five porcine data sets. Figure 6 shows the phantom mounted on its base. The phantom itself is made of flexible silicone $\|$, which as well as being visually realistic enables future work on deformable registration. In order to control the shape of the phantom it is secured in place by a rigid base with 9 mounting pins (Figure 7).

For the porcine cases artificial liver lesions were created using laparoscope guided microwave ablation approximately 1 week prior to resection. After ablation the pigs underwent a contrast enhanced CT scan whilst still insufflated.

The accuracy validation is accomplished by performing a registration as per Section 2 then measuring the system error by comparing the on screen position of visible landmarks with their position as predicted by the model. For the phantom the subsurface mounting pins were used as landmarks. For the porcine data only surface landmarks were available.

\subsection{Data Acquisition}

Each validation data set consisted of a recording of a video sequence and laparoscope tracking data while the scope was moved around the anatomy. Depending on the length and degree of motion involved, a subset of frames (usually every 25 th frame for each channel) and tracking data were extracted from the recorded data. Millisecond time stamping was used to ensure that the tracking and video data were synchronised. Only video frames with tracking data within $20 \mathrm{~ms}$ were used for validation.

For the phantom, the registration (Section 2) is performed using video of the phantom surface. Then the phantom is removed and a validation data set is recorded, filming the mounting pins which serve as subsurface landmarks.

\footnotetext{
${ }{ }_{\text {www.healthcuts.co.uk }}$
} 


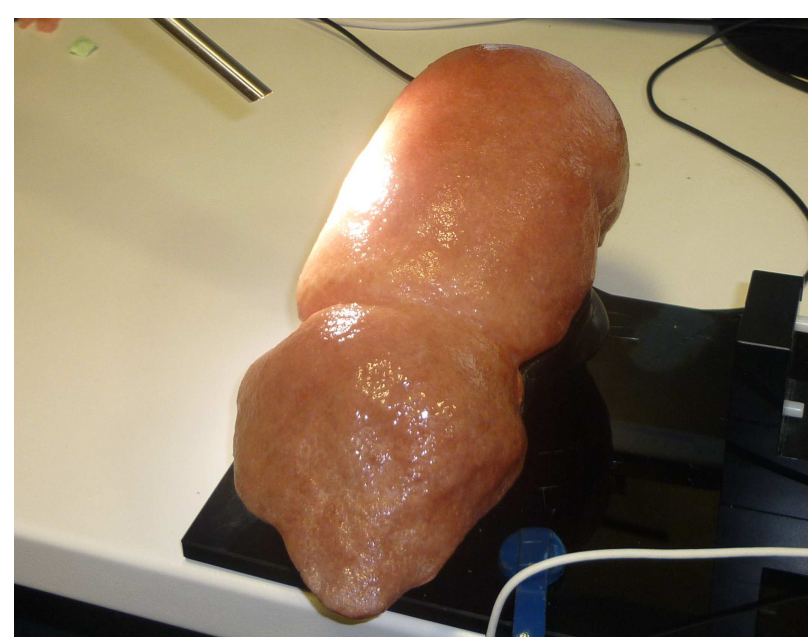

Figure 6 . The silicone phantom mounted on the table in front of the stereo laparoscope. The visible part of the liver phantom is made of visually realistic flexible silicone

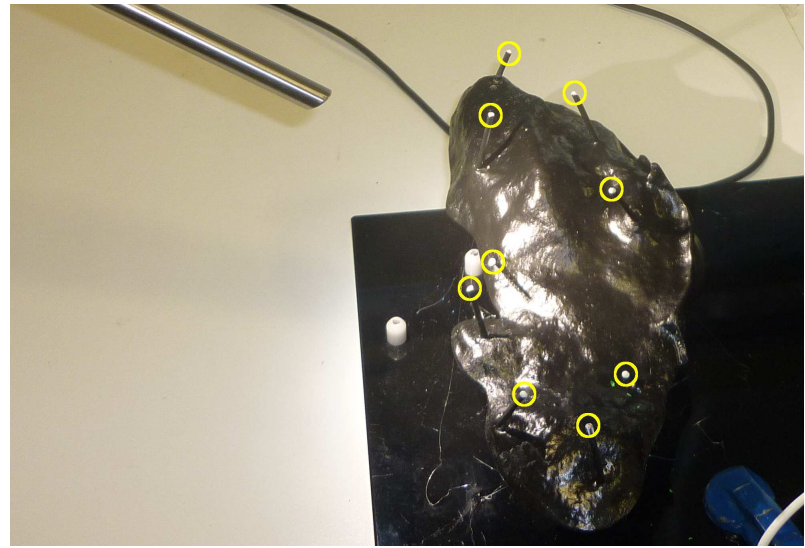

Figure 7. The flexible silicone phantom is mounted on 9 rigid pins (circled) to ensure it maintains its shape in between CT scanning and imaging with the laparoscope. After the silicone phantom is removed the mounting pins are used as subsurface landmarks for the accuracy measurement.

For the in-vivo experiments, subsurface landmarks could not be used, so the landmark points used are on the surface of the liver, e.g. surface ablation zones, and anatomical notches, see Figure 8. As the individual lobes of a porcine liver can move independently, validation was limited to the lobe upon which registration was performed. No respiratory or cardiac gating was used so the in-vivo results include errors due to the motion of the liver, as would be expected in practice. The in-vivo data uses CT data from an insufflated subject.

\subsection{Measuring Gold Standard Feature Projected Positions}

For each frame of video used in validation, landmarks were manually identified. The resultant $2 \mathrm{D}$ pixel locations form the set of gold standard feature positions for each data set. For each data set several hundred point pairs were collected.

\subsection{Estimating Gold Standard Feature Positions in World Coordinates}

The second aim of this paper is to determine the contribution of various system error sources to the overall system error. To enable this an estimate of the position of the gold standard features in world coordinates is useful. Matching stereo pairs of the landmarks were used to triangulate the position of each landmark into lens coordinates. The laparoscope tracking and calibration was then used to transform each point into world coordinates. The triangulation process is noisy due to the narrow baseline of the stereoscope, however we assume the triangulation errors are zero mean so with sufficient frames (several hundred for each data set) triangulation errors should not affect the resulting estimates of gold standard feature positions in intra-operative world coordinates. In any case the average triangulated position for many frames yields a location in world coordinates that minimises the projected error. The difference between the projected location of these gold standard points and the measured gold standard on screen positions can be used to predict the performance of a best case performance for a deformable but intra-operatively static model. The remaining errors will be those due to intra-operative motion, e.g. breathing motion and deformation.

\subsection{Calculating Registered Model Points}

For each data set the landmark points in model coordinates were manually picked in the segmented CT data. The landmark points were transformed to world coordinates using the method detailed in Section 2. The difference between the projected location of these points and the gold standard projected points was used as a measure of the accuracy of the proposed method.

Although the analysis of projected error, including picking the gold standard points on screen, was done after surgery on recorded data, the registrations used were computed in real time during surgery. It is possible 


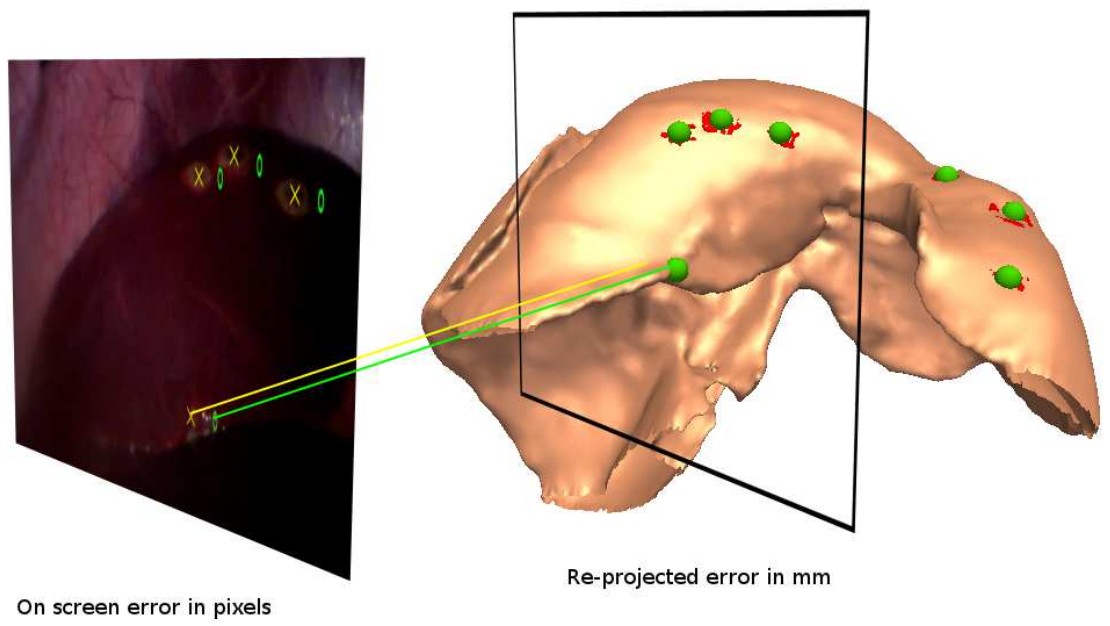

Figure 8. In our porcine experiments, the surface ablations and an anatomical notch were used for error measurement. In the video image, the green circles show the model estimates of the gold standard (yellow crosses) locations. Presenting the errors in pixels does not provide a very useful measure of accuracy, so the errors are re-projected onto a plane parallel to the screen at the depth of the model point. The re-projection errors shown here are around $10 \mathrm{~mm}$ in magnitude.

therefore that a better rigid registration between model and world could be achieved, either with more time spent or with some improvements to the algorithm. To predict the potential accuracy of a system where the registration is properly optimised the following approach was used. The best case rigid registration was assumed to be the rigid body transform that minimises the root mean square (RMS) distance between the landmark points picked in model space and the estimated gold standard landmark points in world coordinates, see section 3.3. This best case rigid registration between two ordered point sets can be found using the "Procrustes" formulation as detailed in Fitzpatrick et al. ${ }^{16}$

\subsection{Error Measure}

Although we use a stereo laparoscope to reconstruct the liver surface, the proposed image guidance system overlays a virtual liver model onto a $2 \mathrm{D}$ screen. Therefore the measure of registration accuracy is based on $2 \mathrm{D}$ on-screen errors in pixels, back-projected to 3D space to convert to millimetre units. Figure 8 shows an example. In the video image, 4 landmark points are visible, and marked using yellow crosses (the "gold standard" feature positions). The same 4 points, identified in the CT scan, are projected onto the video image, here shown as green circles. The difference between the yellow crosses and green circles is a 2D measure of registration error, in pixels. As errors in pixels are of little use in interpreting the size of the errors, the on screen errors are back projected to give errors in mm. For each observed point in the video image, the location is back projected to intersect a plane that is parallel to the screen, and contains the corresponding model point. For each point, the error is measured in this plane, in millimetres. Right and left channels were treated independently.

\section{RESULTS}

The system was tested on a phantom data set and 5 attempted laparoscopic resections of porcine livers. In two of the porcine cases the difference in shape between the pre operative scans and the intra-operative anatomy was so great that no meaningful correspondence could be found despite repeated attempts at registration. On review of the data it appeared that the primary cause of the shape changes was adhesions caused by the ablation process. As such effects should not be present on human cases no further analysis was performed on these data sets.

Registration was achieved during surgery using the method described in section 2, and the usefulness of the system assessed qualitatively. Figure 9 gives two examples of image overlays from the first successful porcine experiment. 

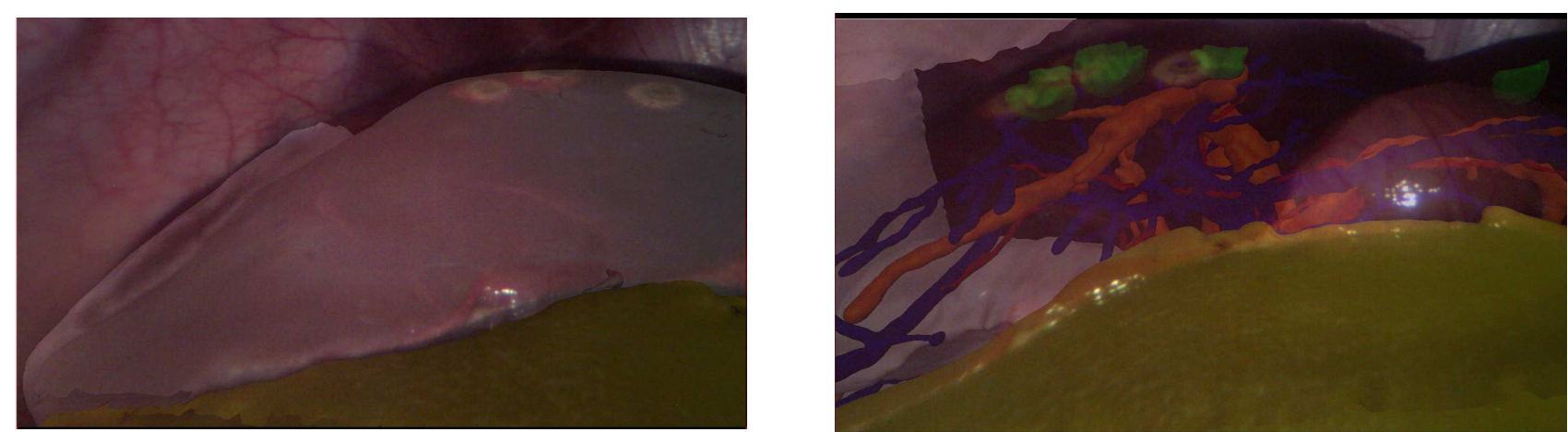

Figure 9. Two examples of image guidance overlays, from the first successful porcine resection (accuracy $8.6 \mathrm{~mm}$ ). On the left only the liver is shown, highlighting reasonable agreement around the edges. At the right vessels and tumours are also shown.

Table 1 summarises the accuracy results for each successful validation experiment, one phantom and 3 porcine data sets. For each experiment the RMS and maximum error are presented along with the number of manually selected gold standard points used in calculation. For each data three registration methods are presented, the best case static deformable registration (Section 3.3), the best case rigid rigid registration, and the proposed method (Section 3.3). The columns of the table indicate the system errors captured by each registration method. Table 1 indicates that the current registration method achieves an accuracy of around $10 \mathrm{~mm}$.

\begin{tabular}{|c|c|c|c|c|c|c|c|c|c|}
\hline Data Set & 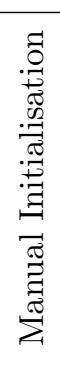 & 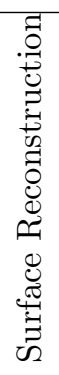 & 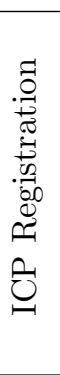 & 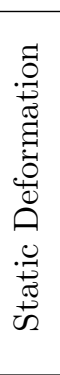 & 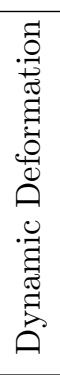 & 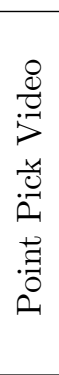 & 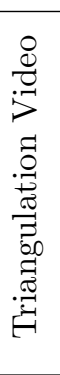 & RMS (mm) & $\operatorname{Max}(\mathrm{mm})$ \\
\hline Phantom Best Static Def. & - & - & - & - & - & - & $\boldsymbol{V}$ & 2.7 & 9.5 \\
\hline Phantom Best Rigid & - & - & - & - & - & $\boldsymbol{v}$ & $\boldsymbol{\nu}$ & 3.3 & 12.5 \\
\hline Phantom Proposed Method & $\checkmark$ & $\checkmark$ & $\checkmark$ & - & - & $\checkmark$ & - & 2.9 & 9.3 \\
\hline Porcine Best Static Def. & - & - & - & - & $\sqrt{V}$ & - & $\checkmark$ & $5.1,6.1,6.5$ & $20.7,26.0,46.7$ \\
\hline Porcine Best Rigid Reg. & - & - & - & $\boldsymbol{v}$ & $\boldsymbol{v}$ & $\boldsymbol{v}$ & $\boldsymbol{v}$ & $7.3,8.2,6.9$ & $20.2,33.4,48.7$ \\
\hline Porcine Proposed Method & $\checkmark$ & $\checkmark$ & $\checkmark$ & $\checkmark$ & $\checkmark$ & $\checkmark$ & - & $8.6,10.7,9.0$ & $18.2,25.6,43.3$ \\
\hline
\end{tabular}

Table 1. The 2 data types and 3 methods used for validation, the system errors captured by each, and results. System errors common to all methods, laparoscope calibration and tracking, and point picking errors in the CT are omitted from the table for clarity. Each data type and method captures a different subset of error sources, shown in the left hand columns. The right hand columns show RMS and maximum errors for each method. Phantom results are calculated from 791 samples of 9 subsurface landmarks. Three sets of results are shown for the porcine data, one for each animal. The number of samples for each porcine data set were, 476 samples of 4 surface landmarks, 234 samples of 6 surface landmarks, and 483 samples of 6 surface landmarks, respectively.

\section{DISCUSSION}

Referring back to the results given in Table 1, the accuracy of around $3 \mathrm{~mm}$ for the phantom data is clinically acceptable. The porcine data provides additional challenges of non-rigid deformation and breathing motion, significantly reducing accuracy. The accuracy required for a useful image guidance system is as yet unknown, Cash et al. ${ }^{7}$ aimed for $10 \mathrm{~mm}$, similar to our results. Current systems in use for open surgery ${ }^{3,17}$ use rigid registration, so will suffer the same problems with intra-operative deformations, so would be expected to achieve accuracies between 7 and $10 \mathrm{~mm}$ (the last two rows of table 1). Anecdotally, the surgeons who used the system 


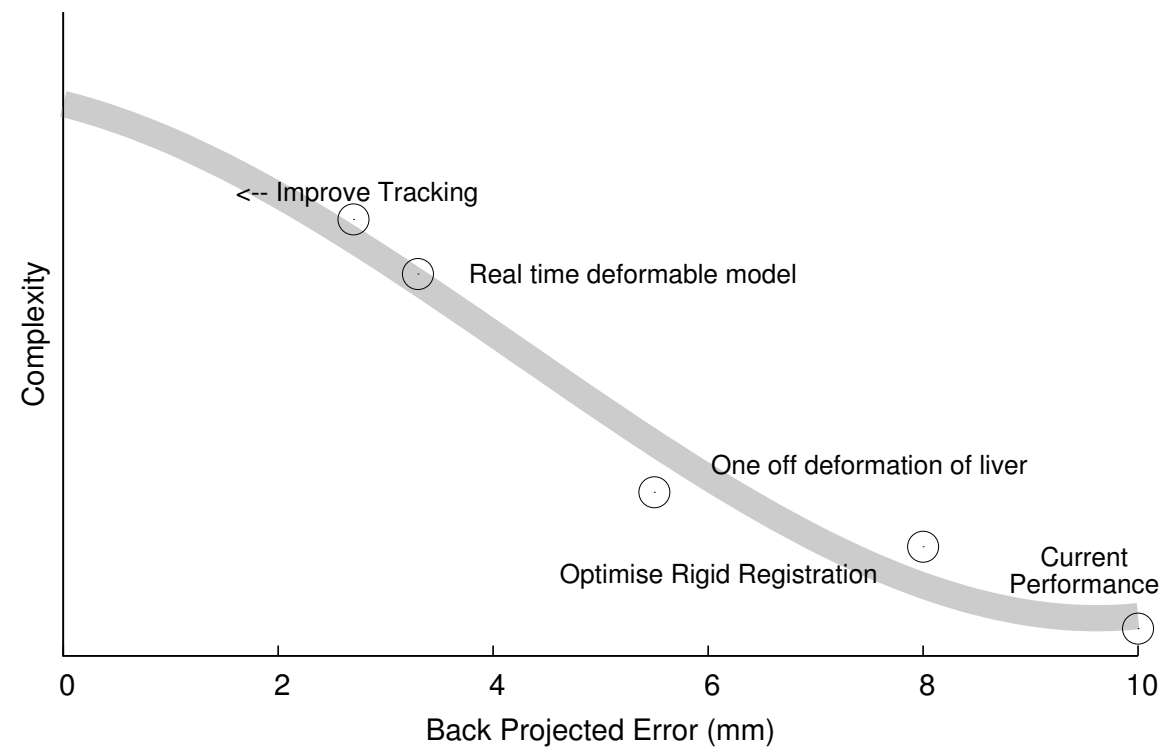

Figure 10. The results from table 1 can be plotted against some measure of "complexity". At present the meaning of this is unquantified.

in-vivo found the overlay technically impressive and useful, but have also stated an accuracy of $3 \mathrm{~mm}$ would be required to ensure adequate margins.

Table 1 can be used to form a development roadmap. Plotting the accuracy figures from table 1 against some measure of system complexity could yield something like Figure 10.

Work is ongoing to improve the registration algorithm and to integrate deformable or locally rigid registration into the system. Early work on human data has indicated that it will be difficult to collect surface patches containing only liver due to the smaller size of the human liver. Current work is therefore focusing on detecting whether the reconstructed surface points are part of the liver, allowing surface points on surrounding anatomy to be removed from the registration. This should enable improvement of the basic rigid registration algorithm. Work is also ongoing on incorporating information from laparoscopic ultrasound into the registration. ${ }^{18}$ With sufficient information it should be possible to use a deformable registration, to this end NiftySim ${ }^{19}$ has been integrated into the NifTK software. The NifTK platform is modular, allowing these and other proposed enhancements to be easily added to the existing work flow and tested. With these improvements it should be possible to achieve accuracies of around $6 \mathrm{~mm}$ (based on "Porcine Best Static Deformation") in the near future. Note that we expect slightly better results on human data, due to tighter constraints on liver motion and less breathing motion, though this is yet to be demonstrated. Achieving accuracy better than this will require either dynamic (primarily breathing) motion models and or improved laparoscope tracking.

The projected error used here effectively removes the errors in the camera normal direction. Whilst this is valid for a $2 \mathrm{D}$ overlays, different visualisations may require $3 \mathrm{D}$ errors to be known. At present, the triangulation errors due to the small distance between the stereo lenses overwhelms the system errors. Work is ongoing to present a meaningful 3D error. The use of insufflated CT data reduces registration errors, but could not be applied to humans. We are currently investigating how to map from non-insufflated CT to the surgical scene inside an insufflated patient.

We have described a working laparoscopic image guidance system for liver resection. The system is novel and capable of performing registration in a sterile setting in around 5 minutes. We propose that clear reporting of numerical errors will provide easier comparison with other systems under development. 


\section{ACKNOWLEDGMENTS}

This publication presents independent research commissioned by the Health Innovation Challenge Fund (HICFT4-317), a parallel funding partnership between the Wellcome Trust and the Department of Health. The views expressed in this publication are those of the authors and not necessarily those of the Wellcome Trust or the Department of Health. The authors also thank NVIDIA for the kindly donated Quadro K5000 and SDI capture card. We also thank CONMED and IRCAD for their support.

\section{REFERENCES}

1. K. Nguyen, J. Marsh, A. Tsung, J. Steel, T. Gamblin, and D. Geller, "Comparative benefits of laparoscopic vs open hepatic resection: A critical appraisal," Archives of Surgery 146(3), pp. 348-356, 2011.

2. T. Kingham, S. Jayaraman, L. Clements, M. Scherer, J. Stefansic, and J. Jarnagin, "Evolution of imageguided liver surgery: transition from open to laparoscopic procedures," J Gastrointest Surg. 17(7), pp. 12741282, July 2013.

3. M. Peterhans, A. vom Berg, B. Dagon, D. Inderbitzin, C. Baur, D. Candinas, and S. Weber, "A navigation system for open liver surgery: design, workflow and first clinical applications," Int J Med Robot. 7(1), pp. 7-16, Mar 2011.

4. T. Igami, Y. Nakamura, T. Hirose, T. Ebata, Y. Yokoyama, G. Sugawara, T. Mizuno, K. Mori, and M. Nagino, "Application of a three-dimensional print of a liver in hepatectomy for small tumors invisible by intraoperative ultrasonography: Preliminary experience," World Journal of Surgery 38(12), pp. 3163-3166, 2014.

5. N. C. Buchs, F. Volonte, F. Pugin, C. Toso, M. Fusaglia, K. Gavaghan, P. E. Majno, M. Peterhans, S. Weber, and P. Morel, "Augmented environments for the targeting of hepatic lesions during image-guided robotic liver surgery," Journal of Surgical Research 184(2), pp. 825-831, 2013.

6. J. Bano, S. Nicolau, A. Hostettler, C. Doignon, J. Marescaux, and L. Soler, "Registration of preoperative liver model for laparoscopic surgery from intraoperative 3d acquisition," in Augmented Reality Environments for Medical Imaging and Computer-Assisted Interventions, H. Liao, C. Linte, K. Masamune, T. Peters, and G. Zheng, eds., Lecture Notes in Computer Science 8090, pp. 201-210, Springer Berlin Heidelberg, 2013.

7. D. M. Cash, M. I. Miga, T. K. Sinha, R. L. Galloway, and W. C. Chapman, "Compensating for intraoperative soft-tissue deformations using incomplete surface data and finite elements," IEEE Transactions on Medical Imaging 24, pp. 1479-1491, Nov. 2005.

8. P. Mountney, J. Fallert, S. Nicolau, L. Soler, and P. W. Mewes, "An augmented reality framework for soft tissue surgery," in Medical Image Computing and Computer-Assisted Intervention-MICCAI 2014, pp. 423431, Springer International Publishing, 2014.

9. M. J. Clarkson, G. Zombori, S. Thompson, J. Totz, Y. Song, M. Espak, S. Johnsen, D. Hawkes, and S. Ourselin, "The niftk software platform for image-guided interventions: platform overview and niftylink messaging," International Journal of Computer Assisted Radiology and Surgery , pp. 1-16, 2014.

10. Z. Zhang, "Flexible camera calibration by viewing a plane from unknown orientations," in The Proceedings of the Seventh IEEE International Conference on Computer Vision, 1, pp. 666-673, 1999.

11. D. Stoyanov, M. Scarzanella, P. Pratt, and G.-Z. Yang, "Real-time stereo reconstruction in robotically assisted minimally invasive surgery," in Medical Image Computing and Computer-Assisted Intervention MICCAI 2010, T. Jiang, N. Navab, J. Pluim, and M. Viergever, eds., Lecture Notes in Computer Science 6361, pp. 275-282, Springer Berlin Heidelberg, 2010.

12. J. Totz, S. Thompson, D. Stoyanov, K. Gurusamy, B. Davidson, D. J. Hawkes, and M. J. Clarkson, "Fast Semi-dense Surface Reconstruction from Stereoscopic Video in Laparoscopic Surgery," in Information Processing in Computer-Assisted Interventions, D. Stoyanov, D. Collins, I. Sakuma, P. Abolmaesumi, and P. Jannin, eds., Lecture Notes in Computer Science 8498, pp. 206-215, Springer International Publishing, 2014.

13. J. D. Stefansic, A. J. Herline, Y. Shyr, W. C. Chapman, J. M. Fitzpatrick, B. M. Dawant, and R. L. Galloway, "Registration of physical space to laparoscopic image space for use in minimally invasive hepatic surgery," IEEE Transactions on Medical Imaging 19, pp. 1012-1023, Oct. 2000. 
14. R. Y. Tsai and R. K. Lenz, "A new technique for fully autonomous and efficient 3d robotics hand/eye calibration," IEEE Transactions on Robotics and Automation, 5(3), pp. 345-358, June 1989.

15. R. B. Rusu and S. Cousins, "3D is here: Point Cloud Library (PCL)," in IEEE International Conference on Robotics and Automation (ICRA), (Shanghai, China), May 9-13 2011.

16. J. M. Fitzpatrick, D. L. G. Hill, and C. R. Maurer Jr, Handbook of Medical Imaging, vol. II, ch. Image Registration. SPIE Press, 2000.

17. J. D. Stefansic, W. A. Bass, S. L. Hartmann, R. A. Beasley, T. K. Sinha, D. M. Cash, A. J. Herline, and R. L. Galloway, "Design and implementation of a pc-based image-guided surgical system.," Comput Methods Programs Biomed 69, pp. 211-224, Nov 2002.

18. Y. Song, J. Totz, S. Thompson, S. Johnsen, D. Barratt, K. Gurusamy, B. Davidson, S. Ourselin, D. Hawkes, and M. J. Clarkson, "Locally Rigid, Vessel Based Registration for Laparoscopic Liver Surgery," in Information Processing in Computer-Assisted Interventions, Lecture Notes in Computer Science, p. In press, Springer International Publishing, 2015.

19. S. Johnsen, Z. Taylor, M. Clarkson, J. Hipwell, M. Modat, B. Eiben, L. Han, Y. Hu, T. Mertzanidou, D. Hawkes, and S. Ourselin, "Niftysim: A gpu-based nonlinear finite element package for simulation of soft tissue biomechanics," International Journal of Computer Assisted Radiology and Surgery, pp. 1-19, 2014. 